\title{
Electroclinical Features of Sleep-Related Head Jerk
}

\author{
Gengyao $\mathrm{Hu}^{1,2, *}$ \\ $\mathrm{Na}$ Yuan $^{\mathrm{l}, *}$ \\ Yuanhang Pan ${ }^{1, *}$ \\ Bi Wang' \\ Xiaoli Wang \\ Zezhi Wang' \\ Ze Chen' \\ Yonghong Liu'
}

'Department of Neurology, Xijing Hospital, Fourth Military Medical University (Air Force Medical University), Xi'an, 7 10032, People's Republic of China; ${ }^{2}$ Department of Neurology, Xi'an People's Hospital (Xi'an Fourth Hospital), Xi'an, 7I006I, People's Republic of China

*These authors contributed equally to this work
Correspondence: Yonghong Liu Department of Neurology, Xijing Hospital, Fourth Military Medical University (Air Force Medical University),

Xi'an, People's Republic of China

Tel +86 I399/236602

Email liuyhong@fmmu.edu.cn
Study Objectives: To evaluate clinical and electrophysiological features of sleep-related head jerk (SRHJ) and electromyographic activity of superficial neck muscles during head jerk.

Methods: Totally, 850 cases with video-polysomnography recording were collected, among which 50 presented with SRHJ. In these 50 patients, 15 underwent electromyography (EMG) check on bilateral sternocleidomastoid (SCM) and trapezius muscles as well as chin, while 35 had only chin EMG check. Further, the sensitivity and specificity of the both EMGs were calculated and compared.

Results: Six among the 50 SRHJ patients had a primary complaint of involuntary head jerks associated with impaired sleep. Approximately $76.1 \%$ of head jerks occurred during REM sleep with the median head jerk index of $5.9 / \mathrm{h}, 64.5 \%$ of which were associated with electroencephalogram arousals and $66.4 \%$ with body movements. One patient showed SRHJ predominantly in NREM sleep but also in wakefulness. Surface EMG of SCM/ trapezius muscles showed a sensitivity of $92 \%$ and a specificity of $97.8 \%$, whereas chin EMG had a sensitivity of $14.5 \%$ and a specificity of $98.8 \%$.

Conclusion: SRHJ was associated with electroencephalogram arousals and might interfere with sleep. Surface EMG of SCM/trapezius muscles exhibited a good accuracy in the revelation of SRHJ.

Keywords: sleep-related head jerk, neck myoclonus, movement disorder, videopolysomnography, electromyography

\section{Introduction}

Sleep-related head jerk (SRHJ) is characterized by sudden myoclonic flexion or version of the head induced by neck muscle contraction, and occurs mainly during rapid eye movement (REM) sleep. In 2010, the motor event termed neck myoclonus was described systematically in 112 patients undergoing polysomnography (PSG), and the authors defined it as a physiological condition because the mean neck myoclonus index was $1 /$ $\mathrm{h}$ REM sleep and only $22 \%$ of neck myoclonus were followed by electroencephalogram (EEG) arousals. ${ }^{1}$ Recently, a few studies indicated that frequent head jerks were related with EEG arousals, which disturbed nighttime sleep and induced excessive daytime sleepiness in some patients. ${ }^{2,3}$ Subsequently, the term "sleep-related head jerk" was substituted for "neck myoclonus" in Wolfensberger's study, ${ }^{2}$ because the former represented more accurately the large head movement even if neck muscles were involved and its duration $(0.5 \mathrm{~ms})$ was longer than that of a typical cortical myoclonus. Meanwhile, this nomenclature is in line with the consensus in the current version of the International Classification of Sleep Disorders that movement disorder is named according to their relationship with sleep. Even with the sleep disturbing feature, SRHJ is still not listed in the latest version of International Classification of Sleep Disorders (ICSD-3). ${ }^{4}$ Whether 
SRHJ is a physiological condition or a sleep-related movement disorder remains a controversial issue.

Neck surface electromyography (EMG) becomes essential in evaluating neck and head activity. Frauscher et al found that $93.8 \%$ of the episodes of neck myoclonus were detected when the electrodes were placed over the bilateral splenius capitis muscles. Moreover, there are over 20 pairs of muscles that control the activity of the neck, and the sternocleidomastoid (SCM) and trapezius muscles are large, easily recognizable, and palpable muscles that are important for the posture of neck and head. ${ }^{5,6}$ It is unknown whether placing the electrodes over the SCM and trapezius muscles is an effective approach for monitoring the head jerk.

In this report, we investigated electroclinical features of SRHJ as well as the potency of neck surface EMG of the SCM and trapezius muscles in determining SRHJ.

\section{Methods}

\section{Population}

Totally, we collected 850 cases with video-PSG recording from January 2020 to December 2020 from the Department of Neurology, Xijing Hospital, Xi'an, China, among which 610 patients underwent chin EMG and 240 had both chin EMG and neck EMG of sternocleidomastoid and trapezius muscles. In these two groups, 35 and 15 respectively showed "stripe-shaped" vertical movement artifacts on the EEG leads and head movements in synchronous video and were diagnosed with SRHJ (Figure 1). The demographic and clinical data of patients were systematically obtained from medical records, including clinical notes, video-PSG reports, and neuroimaging data. The diagnoses of sleep disorder and epilepsy were conducted respectively according to the ICSD-3 criteria ${ }^{4}$ and the International League Against Epilepsy (ILAE) Commission on the definition and classification of epilepsy. ${ }^{7,8}$ This research was approved by the Ethics Committee of Xijing Hospital and was conducted in accordance with the Declaration of Helsinki. Written informed consent was acquired from all participants (from parents or legal guardians of those aged under 18 years).

\section{Polysomnographic Assessment}

All patients underwent one night of 16-hour video-PSG recording in the sleep laboratory with a XLTEK Natus system (USA). Video-PSG included six electroencephalograms (EEG; leads at F3/A2, F4/A1, C3/A2, C4/A1, O1/ $\mathrm{O} 2, \mathrm{O} 2 / \mathrm{A} 1$ ), electro-oculogram, chin electromyogram, and electrocardiogram. Respiration was monitored with a nasal cannula/pressure transducer system, mouth thermistor, chest and abdominal bands, and pulse oximeter. Leg movements in all patients were assessed with surface EMG electrodes placed on the left and right tibialis anterior muscles. Neck muscle activity was evaluated in the 15 patients with additional neck surface EMG over bilateral SCM and trapezius muscles. For upper trapezius, electrodes were placed between the seventh cervical vertebra and the posterior aspect of the acromion along the trapezius muscle line. For SCM, electrodes were attached to the muscle belly of the sternal portion. ${ }^{9}$ The inter-electrode distance was set as $2 \mathrm{~cm}$ (Figure 2). We set the parameters of surface EMG recording based on the international standard with the highpass filter of $10 \mathrm{~Hz}$, low-pass filter of $100 \mathrm{~Hz}$, sampling rate of $500 \mathrm{~Hz}$ and impedance lower than $5 \mathrm{k} \Omega$. Sleep stages, EEG arousals, and legs movements were manually scored

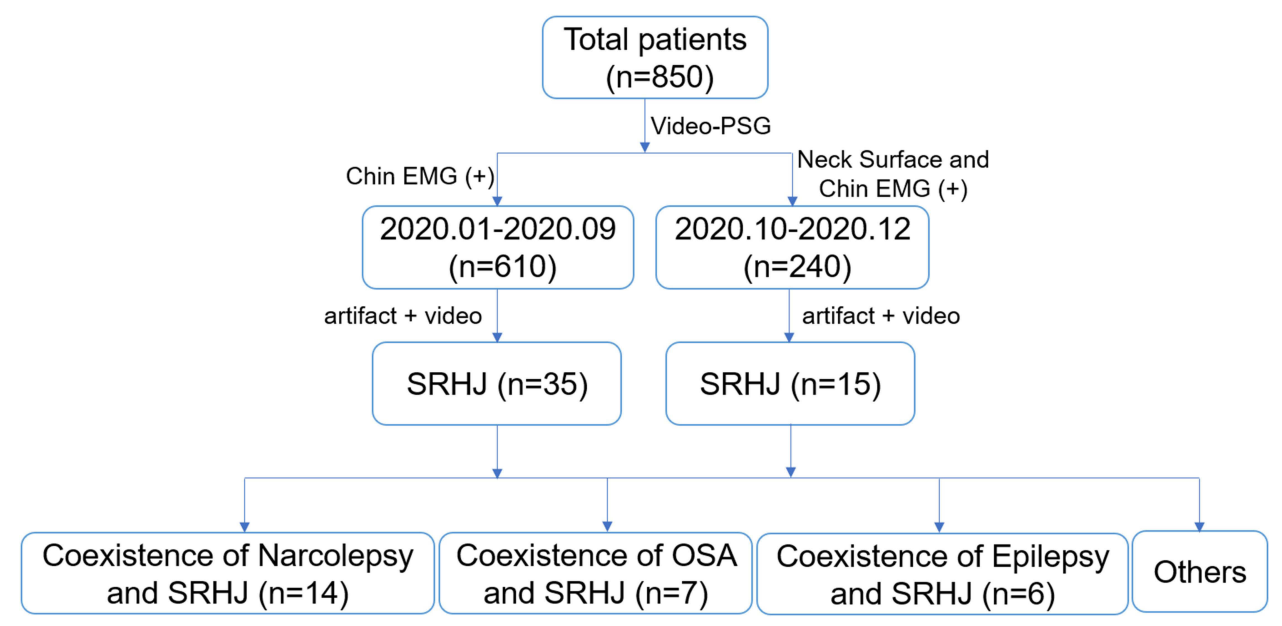

Figure I Flow chart of the study. 

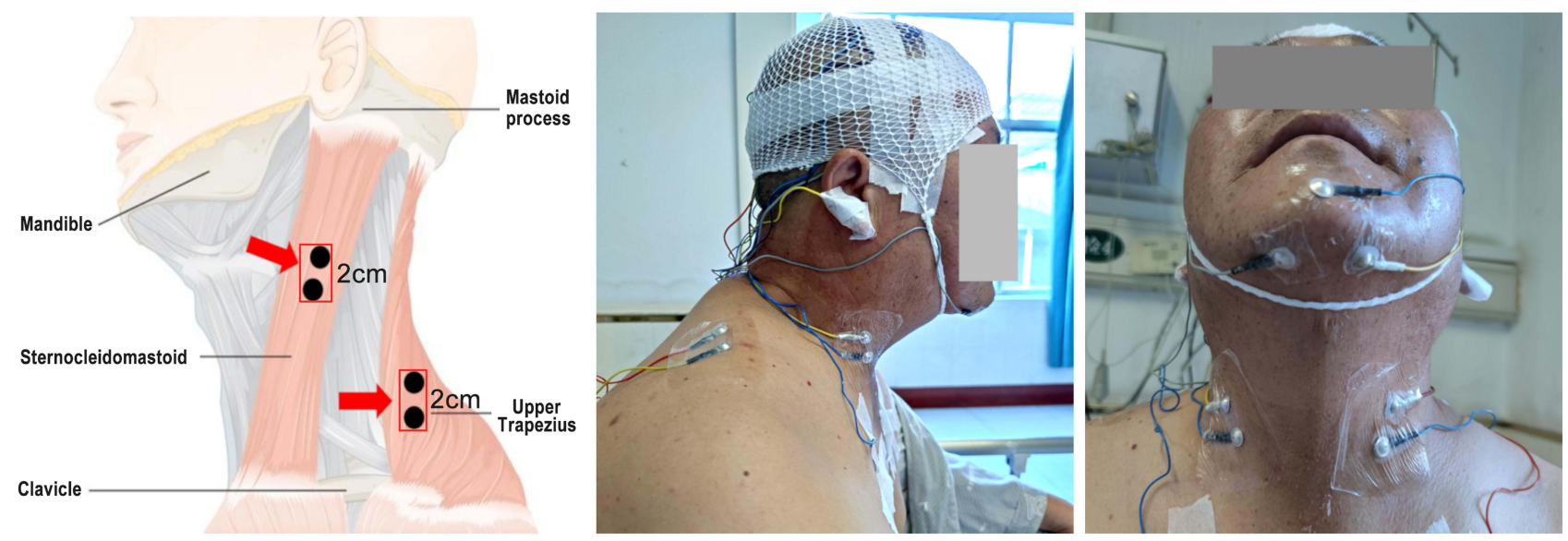

Figure 2 Electrode placement.

according to the American Academy of Sleep Medicine (AASM) manual. ${ }^{10}$

We evaluated head jerk according to the method proposed by Frauscher. ${ }^{1}$ All the video-PSG recordings were carefully investigated by two experienced technologists. Firstly, each 30s epoch was screened for characteristic "stripe-shaped" movement artifacts vertically over the EEG leads (Figures 34). We artificially divided a 30s REM sleep epoch into 10 successive 3s mini-epochs. Each mini-epoch was defined as phasic or tonic REM sleep with the former being defined as the presence of at least one eye movement within a $3 \mathrm{~s}$ mini-epoch, and the latter as the absence of observed eye movement within a 3s mini-epoch. Secondly, the presence of head jerk was inspected from the synchronized video, which was characterized by head movement, including sudden myoclonic dorsal or ventral flexion or turning the head to one side. Differently, head jerks were considered for the analysis even if they were followed by movement-induced EEG artifacts lasting $>2 \mathrm{~s}$. We excluded epileptiform discharges and arousal-inducing head movements as SRHJ is defined as a non-epileptic head jerk. We

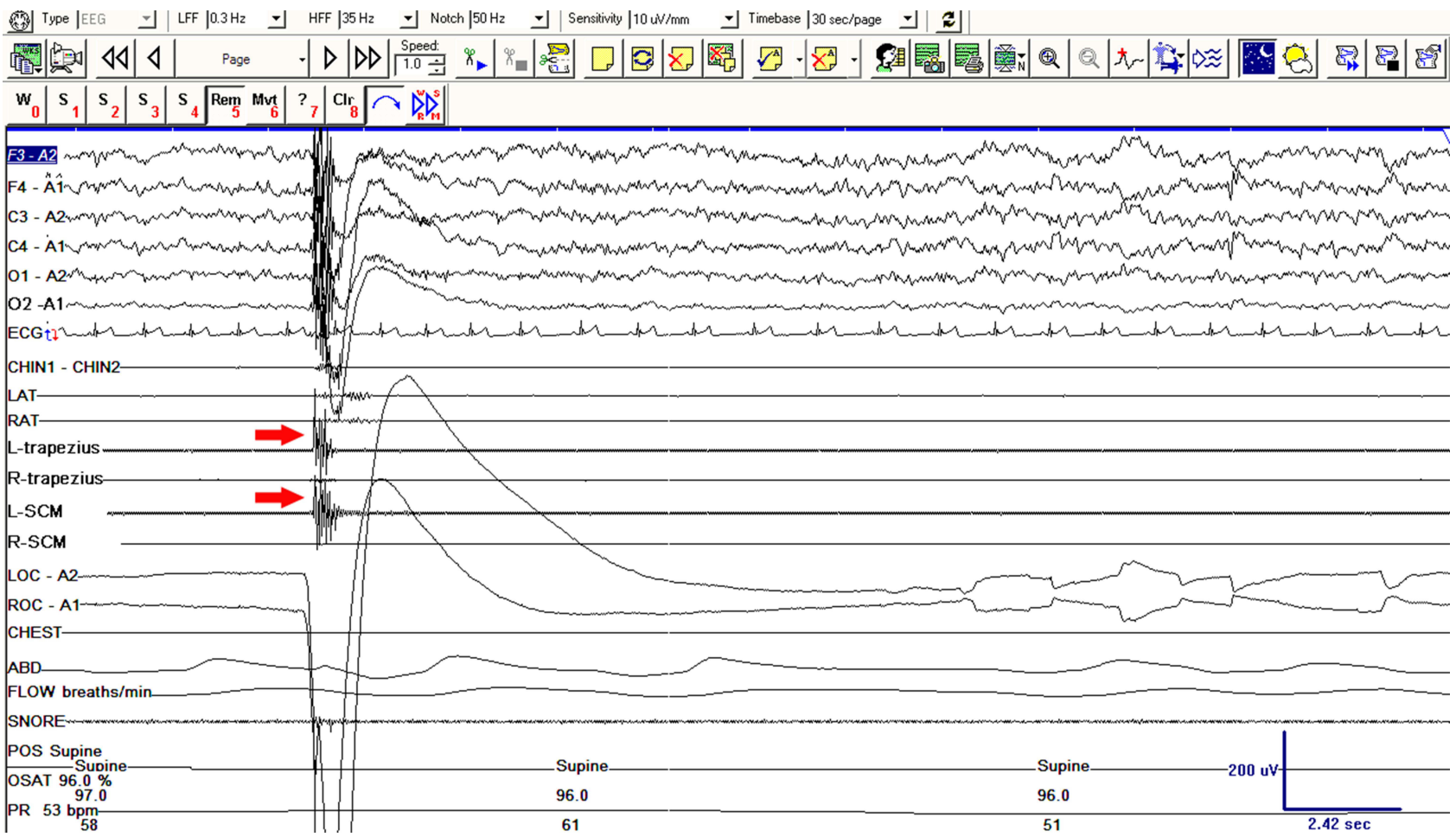

Figure 3 Polysomnographic characteristics of SRHJ during REM sleep in patient II. Red arrows indicate EMG bursts. 


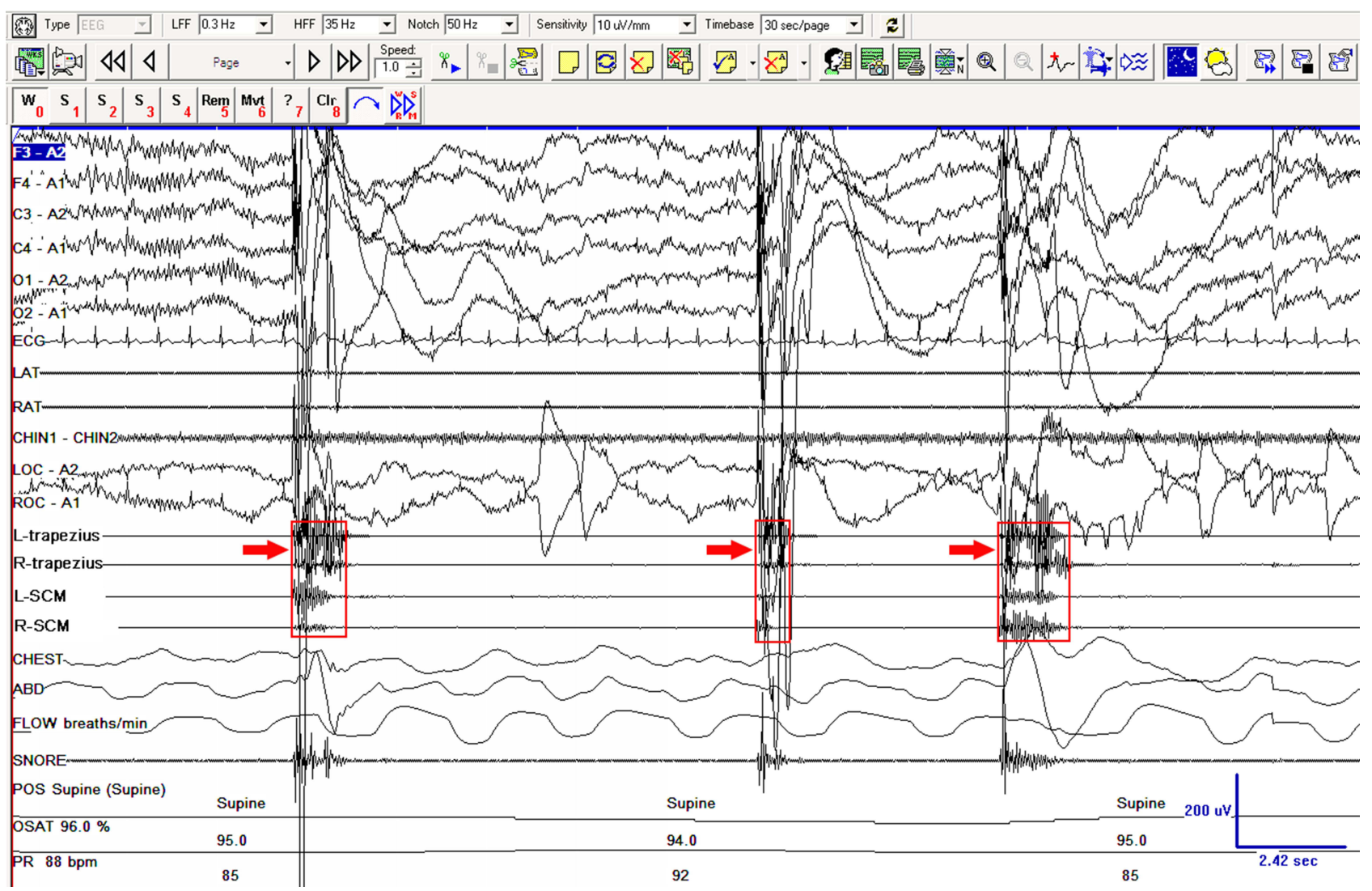

Figure 4 Polysomnographic characteristics of SRHJ during wakefulness in patient 25. Red arrows indicate EMG bursts.

calculated the index of head jerk, defined as the sum of head jerks per hour of total sleep, per hour of NREM (non-REM) sleep, or per hour of REM sleep (including tonic and phasic REM sleep). Furthermore, we identified all events in the video recording from the beginning to the end which included not only SRHJ but other movements. Then the sensitivity and specificity of neck muscles and chin EMG for the diagnosis of SRHJ were calculated respectively. Specifically, the sensitivity was defined as the numbers of SRHJ with EMG activation in the whole SRHJ episodes and the specificity as the numbers of non-SRHJ without EMG activation in the whole non-SRHJ episodes. SRHJ appeared as either isolated or clustered type with the former accompanying with a definite EMG burst, the latter was not. The isolated head jerk was applied for analysis of duration of the EMG burst. The concomitant EEG arousals, awakenings, stage shifts, and body movements were assessed in all patients.

\section{Statistical Analysis}

Data were expressed as median (lower quartile, upper quartile) for skewed data, and mean (standard deviation) for data with normal distribution. Student's $t$-test, Mann-Whitney
$U$-test and chi-square test were used for statistical analysis, as appropriate. All tests were two-sided, and $p$-values $<0.05$ were considered to indicate statistical significance. All the statistical analyses were performed using the IBM SPSS 19.0 software.

\section{Results}

\section{Cohort Characteristics}

In a total of 850 patients, $37.2 \%$ were diagnosed with obstructive sleep apnea, 32\% with insomnia, $8.6 \%$ with narcolepsy, $2.7 \%$ with periodic leg movements in sleep, $2.7 \%$ with REM sleep behavior disorder, and the rest with other sleep disorders. There were 50 patients having SRHJ with a mean age of $27.2+15.1$ years (range, 3-62 years), among whom 36 (72\%) were male. Twenty-two pediatric patients aged 18 years and less. Six patients complained of involuntary head movements associated with impaired sleep but showed a normal brain MRI and family history. Three patients (Patients 23, 24, and 25) with the age of 50-54 years, reported seriously impaired sleep, daytime functioning and emotional stability due to frequent head jerks occurring not only in sleep but also wakefulness. 
They could not fall asleep quickly, as the motor events occurred frequently when they lay down on the bed and felt drowsy during the day and at night. However, the events would disappear when the patients changed to a sitting position. The insomnia in these patients was mainly due to SRHJ. In addition, periodic leg movements in sleep might contribute to the insomnia of patients 23 and obstructive sleep apnoea related to that of patient 25 . Patient 25 also reported the hot weather as a worsening factor for head jerks, which had lasted for 3 years when he was admitted to our clinic to receive his first PSG monitoring. He was readmitted six months later (about 42 months from the first episode) for aggravated conditions. His head jerks were substantially reduced after clonazepam ( $2 \mathrm{mg} / \mathrm{night})$ treatment, recurred following drug withdrawal and eased with the resume of clonazepam. The other two patients refused to take clonazepam exhibiting persistent head jerks and poor sleep for 3-4 years. The diagnoses of the cohort, comorbidities, and medications were reported by two experienced sleep and epilepsy experts, which are presented in Table 1. Among the 50 SRHJ patients, 14 (28\%) were diagnosed with narcolepsy, 7 (14\%) with obstructive sleep apnea, 6 (12\%) with epilepsy, and less than half with other sleep disorders.

\section{Assessment of Head Jerk}

A total of 1168 episodes of head jerk were recorded during the whole night PSG, and the median number of head jerks and median head jerk index for each patient were 12 (6, $20)$ and $1.6(0.8,3.3)$, respectively. In the six patients who complained of head movement associated with impaired sleep, the head jerk index and proportion of associated EEG arousals were higher than that of the other 44 patients $(\mathrm{p}<0.05)$.

During REM sleep, 889 (76.1\%) episodes were recorded with the median head jerk index of 5.9 (2.4, 10.3). The proportion of the motor events occurring sequentially in the first, second, third, fourth, fifth, and sixth REM period were $13.2 \%, 25.4 \%, 29.2 \%, 11.6 \%$, $20 \%, 0.7 \%$, respectively. The events were almost equally distributed between phasic (58.5\%) and tonic REM sleep (41.5\%), and no statistical difference of SRHJ index between the two phases $(p>0.05)$ was observed. Additionally, 573 (64.5\%) episodes of head jerk were associated with EEG arousals, among which five transitioned to NREM sleep and five transitioned to full awakenings, and $590(66.4 \%)$ were associated with body movements.
During NREM sleep, 247 (21.1\%) episodes of head jerk were recorded, most of which occurred within a few minutes preceding or following the adjacent REM period. The median head jerk index of NREM sleep was $0.2(0.0$, $0.6)$, which was significantly lower than that of REM sleep $(p<0.05)$. Approximately $124(50.2 \%)$ occurred in NREM stage 1, 108 (43.7\%) in NREM stage 2, and $15(6.1 \%)$ in NREM stage 3. Surprisingly, 208 (89\%) were associated with EEG arousals, among which four transitioned to NREM 1 sleep and seven transitioned to full awakenings, and $150(60.7 \%)$ were associated with body movements.

In the three patients showing head jerks in both sleep and wakefulness, the SRHJ numbers were 141 and 25 respectively in NREM and REM sleep. All of the 32 episodes in wakefulness occurred after a spontaneous awakening from NREM 1 sleep. The mean duration of alpha rhythm background before and after SRHJ were 182s (range, 2-482s) and 247.7 s (range, 8-1226s). It always occurred abruptly in clusters, consisting of two or more consecutive head movements (Supplementary Video). Of the total 198 episodes in these three patients, $152(76.8 \%)$ were associated with EEG arousals and 167 (84.3\%) with body movements.

\section{Detection of Head Jerk in Neck Surface EMG}

Totally, 339 episodes of head jerk were observed in 15 patients. Neck surface EMG of the SCM/trapezius muscles showed a sensitivity of $92 \%$ and a specificity of $97.8 \%$, whereas chin EMG had a sensitivity of $14.5 \%$ and a specificity of $98.8 \%$. The median EMG duration of $\mathrm{SCM} /$ trapezius muscles was $300 \mathrm{~ms}(200,400)$, with no statistical difference between wakefulness, NREM sleep, and REM sleep ( $>0.05)$, and the one recorded by chin muscle was $300 \mathrm{~ms}(275,400)$. Different from the activation of SCM/trapezius muscles which occurred mainly in REM sleep, the chin muscle activation distributed equally in both REM and NREM sleeps. EMG duration of the $\mathrm{SCM}$ /trapezius muscles in adult patients was slightly longer than that of pediatric patients $(\mathrm{p}<0.05)$.

\section{Discussion}

In the present study, we investigated the electroclinical features of SRHJ and found the SRHJ was associated with EEG arousals in both REM and NREM sleep and, thus, might potentially interfere with sleep. The surface EMG of the SCM/trapezius muscles acted as a new approach with high sensitivity and specificity for SRHJ 


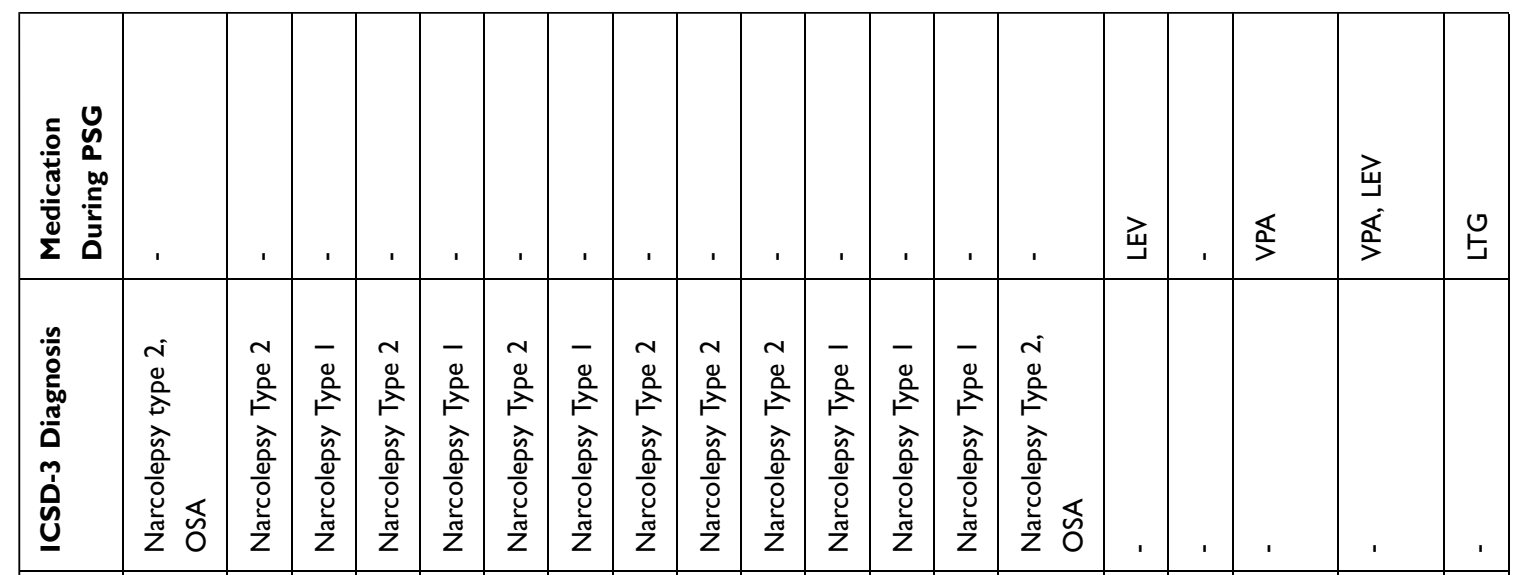

\begin{tabular}{|c|c|c|c|c|c|c|c|c|c|c|c|c|c|c|c|c|c|c|}
\hline 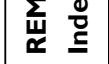 & $\underline{a}$ & $\underline{\underline{n}}$ & นุ & $\stackrel{\dot{m}}{m}$ & $\stackrel{⿱ 亠 幺}{\sim}$ & ó & ఫै & $\stackrel{\text { i }}{\mathrm{N}}$ & $\stackrel{m}{i}$ & $\stackrel{\infty}{m}$ & 우. & $\sim$ & $\stackrel{\infty}{=}$ & นุ & $\stackrel{m}{\sim}$ & $\hat{\mathbf{N}}$ & ก่ำ & $\overline{\dot{r}}$ \\
\hline
\end{tabular}

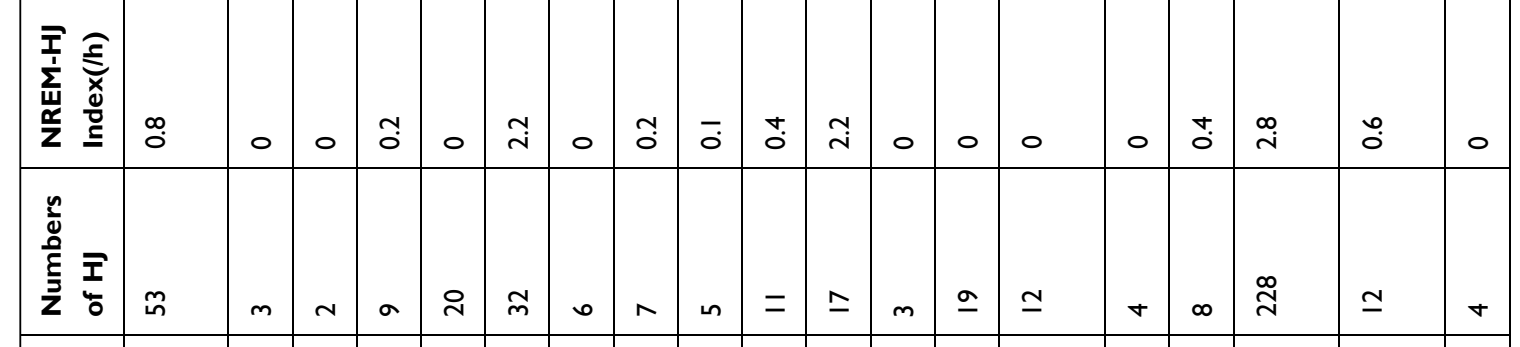

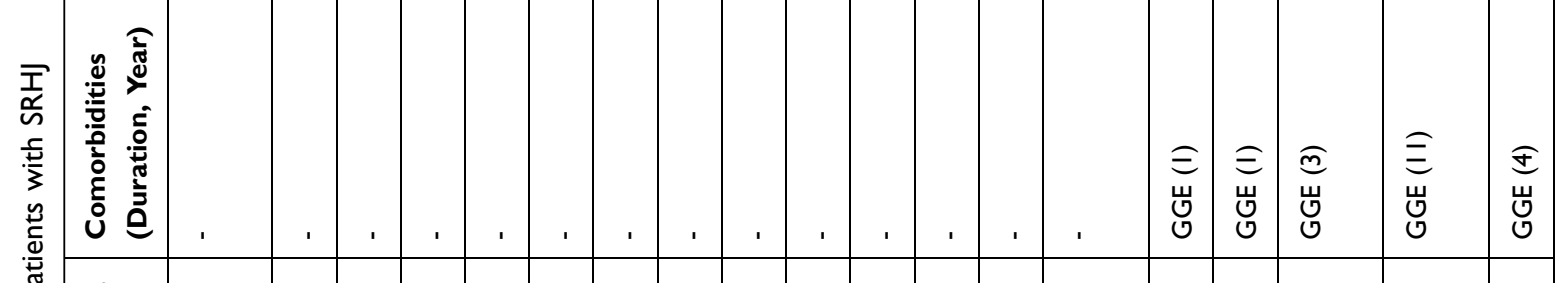

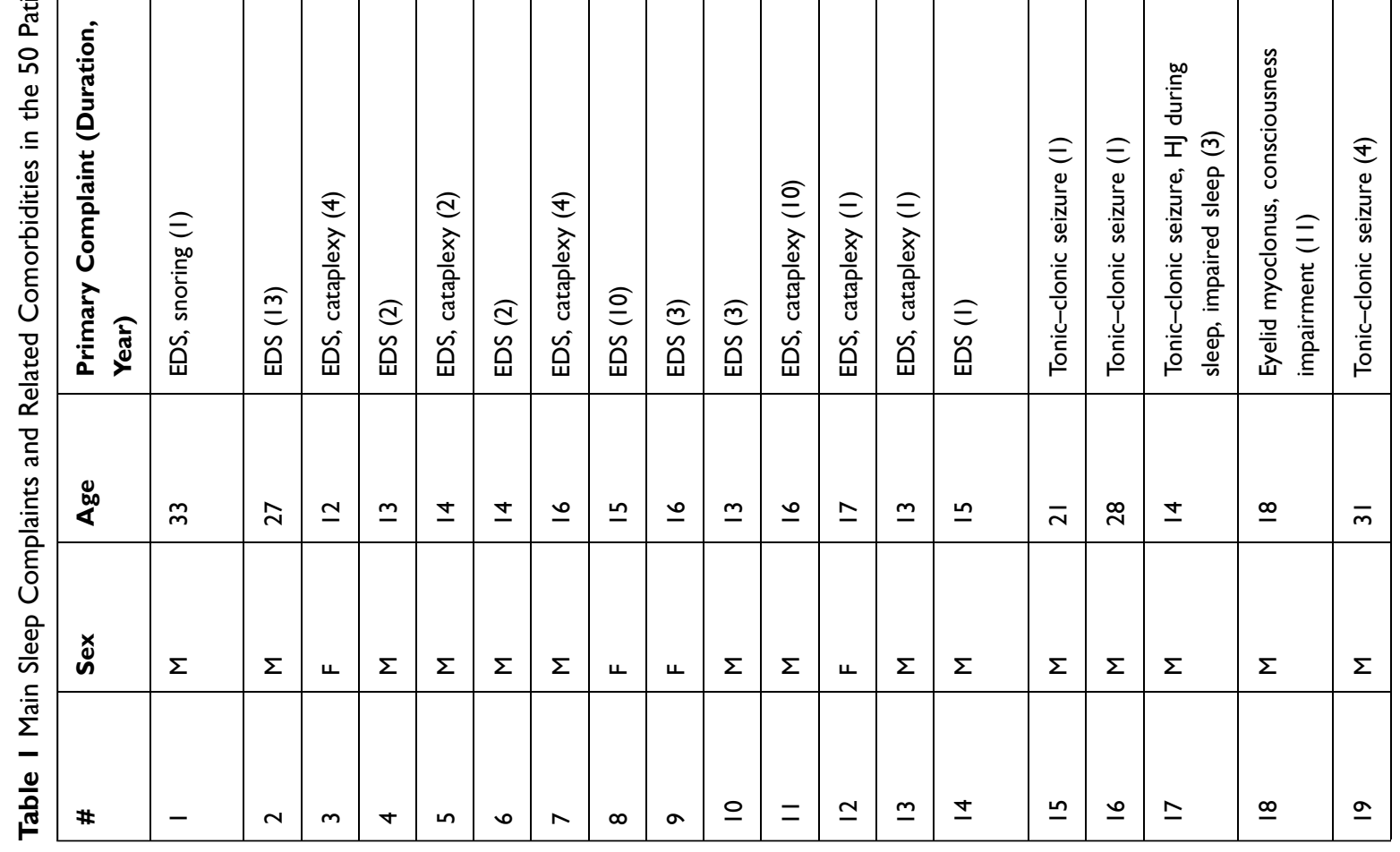




\begin{tabular}{|c|c|c|c|c|c|c|c|c|c|c|c|c|c|c|c|c|c|c|c|}
\hline 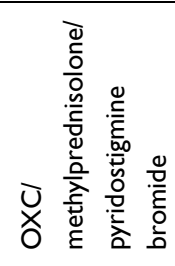 & , & ' & & ' & ' & ' & ' & ' & ' & ' & . & & & 1 & ' & & ' & & ' \\
\hline ' & ' & ' & $\sum_{\square}^{n}$ & ' & 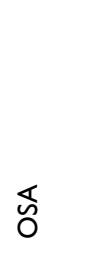 & 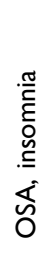 & 菸 & 范 & 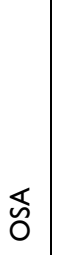 & 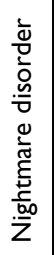 & 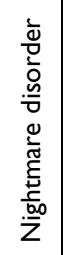 & 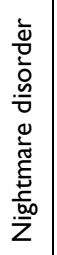 & 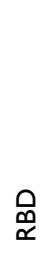 & 怘 & 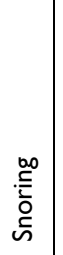 & 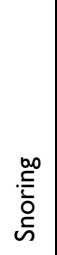 & 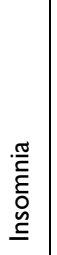 & 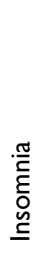 & $\sum_{a}^{\infty}$ \\
\hline$\underline{m}$ & $\grave{\sim}$ & $\stackrel{\infty}{\stackrel{\infty}{0}}$ & $\stackrel{0}{\infty}$ & $\stackrel{+}{i}$ & $\stackrel{\stackrel{m}{\underline{n}}}{\underline{n}}$ & $\bar{i}$ & $\hat{\sigma}$ & $\stackrel{+}{\mathrm{d}}$ & $\overline{\underline{\alpha}}$ & $\stackrel{\square}{ \pm}$ & $\stackrel{n}{n}$ & $\stackrel{m}{\sim}$ & $\stackrel{\ddot{\omega}}{\sim}$ & $\Xi$ & $\overline{\text { in }}$ & $\underset{\infty}{+}$ & $\stackrel{\circ}{i}$ & $\stackrel{ \pm}{=}$ & $\underset{m}{\tilde{m}}$ \\
\hline 0 & 0 & - & $\underline{\text { 누 }}$ & $=$ & $\overline{\mathrm{i}}$ & $\stackrel{m}{0}$ & 0 & 0 & $\stackrel{t}{0}$ & ชี & $\stackrel{\circ}{0}$ & $\stackrel{a}{0}$ & 0 & $\stackrel{\circ}{\longrightarrow}$ & $\stackrel{\circ}{\circ}$ & กั & ז̃ & $\ddot{0}$ & $\stackrel{n}{\circ}$ \\
\hline$N$ & $\stackrel{\sim}{\sim}$ & $\hat{\sim}$ & 이 & $\underline{m}$ & $\stackrel{\circ}{\circ}$ & 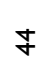 & $\infty$ & $\nabla$ & $\hat{m}$ & in & $\wedge$ & $\sigma$ & : & $\wedge$ & $\simeq$ & \pm & 0 & $\underline{\sigma}$ & N \\
\hline 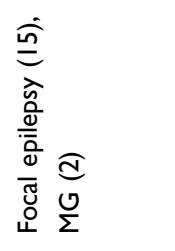 & & ' & & . & 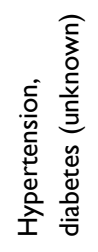 & . &. & . & , & , & . & , & 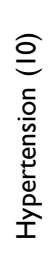 & $\begin{array}{l}\widehat{D} \\
\overleftarrow{\Delta} \\
\Sigma\end{array}$ & . & & , & & . \\
\hline 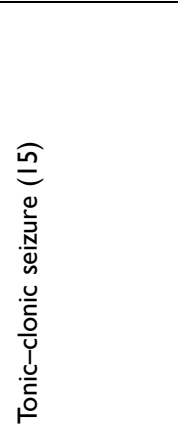 & 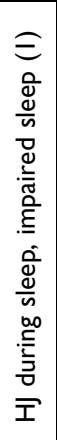 & 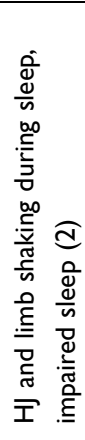 & 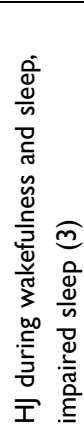 & 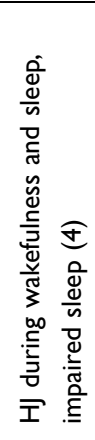 & 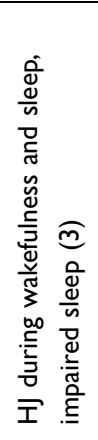 & 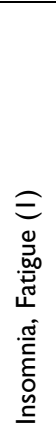 & 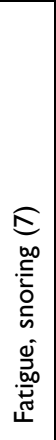 & 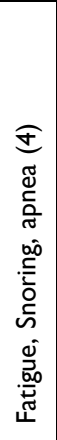 & 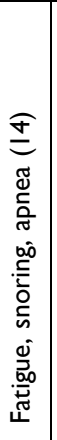 & 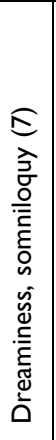 & 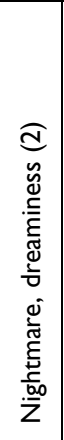 & 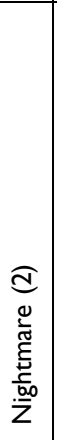 & 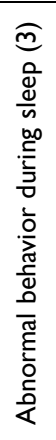 & 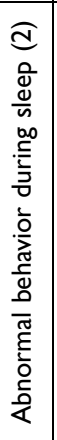 & 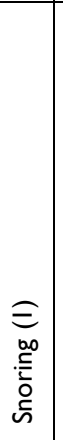 & 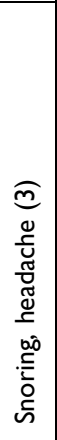 & 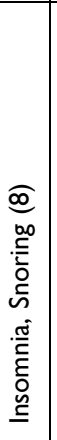 & 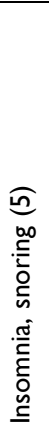 & 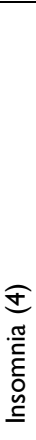 \\
\hline$\underline{\infty}$ & $\stackrel{\infty}{\sim}$ & 느 & 苛 & 운 & $\bar{n}$ & กี & $m$ & ப் & $\bar{m}$ & ㅇ & o & ㅇ & in & in & \pm & f & $\widetilde{\sigma}$ & ᄋ & $\stackrel{\infty}{\sim}$ \\
\hline 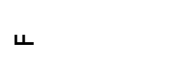 & $\Sigma$ & $\Sigma$ & 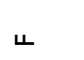 & ч & $\Sigma$ & $\Sigma$ & ч & $\Sigma$ & $\Sigma$ & 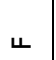 & $\Sigma$ & $\Sigma$ & $\Sigma$ & $\Sigma$ & $\Sigma$ & $\Sigma$ & น & $\Sigma$ & $\Sigma$ \\
\hline ㅇ & $\bar{N}$ & ה & $\tilde{N}$ & $\overleftarrow{\sim}$ & $\stackrel{\sim}{\sim}$ & $\stackrel{\sim}{\sim}$ & $\lambda$ & $\stackrel{\infty}{\sim}$ & సి & ஓ & $\bar{m}$ & $\tilde{m}$ & $m$ & $\dot{m}$ & $\stackrel{\text { m }}{\text { r }}$ & $\stackrel{m}{m}$ & $\hat{m}$ & $\stackrel{\infty}{m}$ & ले \\
\hline
\end{tabular}




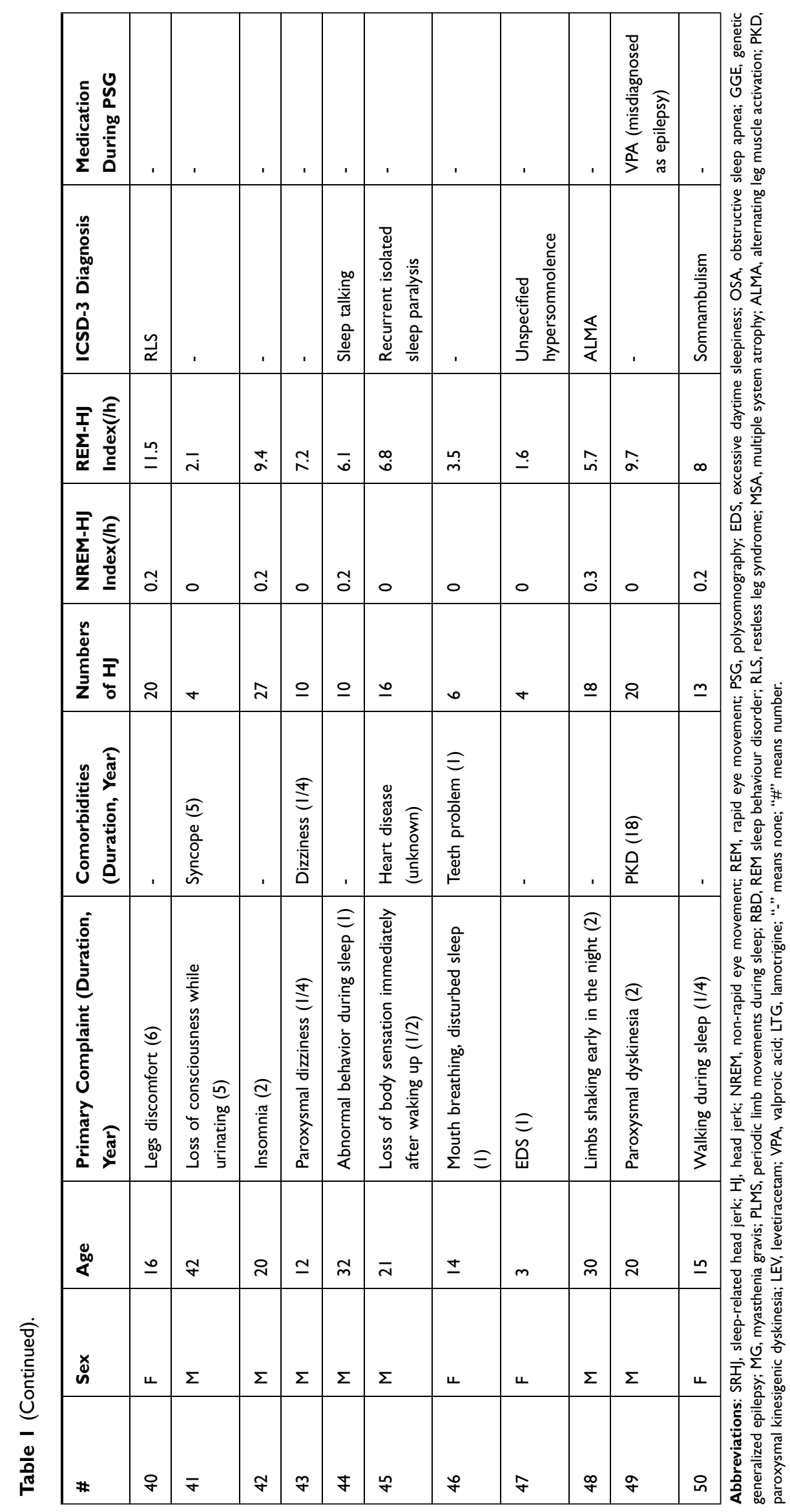


recognition, which would be useful for cases with atypical artifacts or absence of video.

In our study, the head jerk index of REM sleep was 5.9 episodes per hour, which was higher than that of 1.0 in a previous study. ${ }^{1}$ This discrepancy might be due to the differences in methodology. Frauscher et al excluded all SRHJ events followed by an artifact from the analysis, and therefore lost most of the events associated with a significant arousal or body movements. One study reported that the sleep associated breathing disorders accounted for $67.9 \%$ of the 112 patients with neck myoclonus (SRHJ). ${ }^{1}$ In the present study, SRHJ often occurred with narcolepsy, obstructive sleep apnea, epilepsy, and other sleep disorders. SRHJ has ever been shown in a few young patients with type 2 narcolepsy, ${ }^{2,3}$ which was also demonstrated in the present study, but the difference between type 1 and type 2 narcolepsy was insignificant. Narcolepsy is a unique sleep disorder characterized by excessive daytime sleepiness and disrupted nighttime sleep, which is caused by defective REM regulation. The above information indicates that a common pathogenesis of REM dysfunction may play a role in the development of both narcolepsy and SRHJ. The potential significance and pathophysiologic mechanism of the condition is still unclear and warrants further clinical and basic researches.

Previous studies have demonstrated that the SRHJ was a REM sleep-related movement associated with EEG arousals, varying from $20 \%$ to $65.2 \%$ of episodes of head jerk, which was appeared more frequently in younger individuals. ${ }^{1-3} \mathrm{We}$ confirmed these findings, as $76.1 \%$ of head jerks occurred during REM sleep and approximately two-thirds of head jerks were accompanied by EEG arousals. Interestingly, in the three middle-aged patients who had serious sleep disorders, daytime dysfunction, and emotional instability caused by SRHJ, PSG detected most head movements during NREM sleep and wakefulness. On average, each episode consisted of two or more consecutive clusters of head jerks, which was distinct from sleep-related rhythmic movements as it was nonrhythmic jerk of brief duration. ${ }^{11,12}$ Our results indicate that there may be a new characteristic phenotype of SRHJ with predominance in NREM sleep and wakefulness. We understand that the wake-related episodes may not be fully conform to the diagnosis of "sleep-related head jerk"; however, it constitutes a rare condition, as in this study only one patient exhibited the feature in PSG recording. There are similar situations in the naming of neurological events such as the periodic leg movements during sleep (PLMS) which occasionally also appears in wakefulness. ${ }^{13} \mathrm{SRHJ}$ is quite a new observation, which has been termed as "SRHJ" or "neck myoclonus" in different reports, for which further studies concerning the particular clinical and electroclinical feature of patients of this type are warranted.

Myoclonus is a hyperkinetic movement characterized by sudden, brief, involuntary jerks of a single muscle or a group of muscles, which can be classified into four subtypes involving cortical, subcortical, spinal (segmental and propriospinal), and peripheral forms according to anatomy origin. ${ }^{14,15}$ In line with previous studies, the duration of EMG burst in our study was significantly longer than cortical myoclonus. Moreover, Wolfensberger et al reported neither EEG abnormalities nor a Bereitschaftspotential calculated by a back-averaging analysis in SRHJ events, precluding the involvement of cortical activation. $^{2}$ Although the underlying pathophysiology of SRHJ remains poorly understood, it has been hypothesized that glycinergic dysfunction may play a role. ${ }^{3}$ Glycinergic and GABAergic dysfunctions in transgenic mice with a mutant glycine receptor trigger features of REM sleep behavior disorder as well as head and limb jerks during REM sleep. ${ }^{16}$ In our study, clonazepam, a long GABA receptor agonist, was reported to suppress the head jerks and improve sleep in one patient. Similarly, Frauscher et al reported 13 patients who were administered benzodiazepine medication and experienced no head jerk, but the authors claimed that there was no correlation between benzodiazepine administration and the absence of SRHJ, as clonazepam was reported to have no or slight effect on EMG activity of periodic leg movements but to increase sleep stability. ${ }^{17}$ The importance of disinhibition of GABAergic systems in myoclonus has been notified, as the disruption of $\mathrm{GABA}_{\mathrm{A}}$ receptor in the neural axis produces clear myoclonus. ${ }^{18}$ Thus, it is speculated that the SRHJ may be correlated with GABAergic dysfunction, which increases neuronal excitability in subcortical or downstream areas of the neural axis.

Whether SRHJ is a physiological or pathological event remains a controversial issue. The former viewpoint was established on the findings that the head jerk occurred in $50 \%$ of the subjects without frequent associations with EEG arousals. ${ }^{1}$ However, a recent study involving a series of 30 subjects with sleep disorders and/or sleep-related head jerks and receiving PSG monitoring disclosed excessive SRHJ (defined by head jerk $>30$ /hour) in REM sleep, suggesting it may be a pathological movement disorder in some patients. ${ }^{4}$ During REM sleep, the occurrence of peripheral muscle twitches and rapid eye movement are present in phasic sleep but not in tonic sleep. However, the present study demonstrated no statistical difference in the distribution of SRHJ and SRHJ index between tonic and phasic REM sleeps. This was in line with the study of 
Wolfensberger et $\mathrm{al}^{2}$ who did not find a significant association between SRHJ and ocular movements in REM sleep. Thus, it is probable that SRHJ might not be an exaggeration of the physiological twitches. Our study included six patients with a primary complaint of SRHJ associated with impaired sleep and 44 patients being unaware of head movements with uncertain clinical significance. Similar to the term "sleep-related rhythmic movement and rhythmic movement disorder" raised by Prihodova et al, ${ }^{11}$ we propose that SRHJ should be classified as either a normal or a pathological condition based on frequency, intensity and comorbidity.

Neck surface EMG is important for identifying SRHJ. Neck EMG of bilateral splenius capitis was activated in $93.8 \%(61 / 65)$ of head jerks. ${ }^{1}$ The splenius capitis is a large muscle involved in head rotation and neck extension, and much of it lies deep to the trapezius and only a portion of it runs close to the surface. There are a number of other muscles, including the SCM, upper trapezius, and semispinalis capitis, close to the surface portion of the splenius capitis. It is important to confirm that the surface EMG data originates directly from the splenius capitis and is not influenced by interference from the adjacent musculature. Compared with the splenius capitis, the SCM and trapezius muscles are more easily recognized that play a crucial role in the posture of the neck and head. ${ }^{5,6}$ In our study, we confirmed that recording of SCM and trapezius muscles were better and more specific for SRHJ recognition than recording of chin muscle. Combined with the findings of a previous study, ${ }^{1}$ we postulate that SRHJ is a large muscle group movement involving SCM, trapezius, and splenius capitis muscles (except for chin muscles that are not critical for posture of head and neck).

There were some limitations in this study. Firstly, our study population was small and came from a single center, which focused mainly on patients with epilepsy and sleep disorders. This prevented us from understanding the cooccurrence of other diseases with SRHJ. Secondly, the patients in our study only received one night of sleep monitoring, and the night-to-night variability was not measured. However, Frauscher et al found no significant difference in the night-to-night variability of SRHJ index. ${ }^{1}$ Thirdly, Pittsburgh Sleep Quality Index and Epworth sleepiness scale were not performed to assess the sleep quality and daytime functioning. Further studies with large population are warranted for identifying the cut-off of SRHJ between physiological and pathological conditions, and evaluating the effectiveness of clonazepam on SRHJ.

\section{Conclusions}

The results of the current study suggest that, although SRHJ accounted for sleep disorder in relatively few patients, it was significantly associated with EEG arousals and might seriously impair sleep and daytime functioning, which should be paid more attention and if possible, this condition should be included in the ICSD-3. Furthermore, we identified surface EMG of the SCM/trapezius muscles as a new method for SRHJ recognition, which may be especially useful for cases with atypical artifacts or absence of video.

\section{Acknowledgment}

We declare that the work described herein was an original research that has not been published previously, and not under consideration for publication elsewhere, in whole or in part.

\section{Disclosure}

Dr Yonghong Liu reports grants (No. 2019ZTB03, 2020JSTS21) from Air Force Medical University. The authors report no other conflicts of interest in this work.

\section{References}

1. Frauscher B, Brandauer E, Gschliesser V, et al. A descriptive analysis of neck myoclonus during routine polysomnography. Sleep. 2010;33:1091-1096. doi:10.1093/sleep/33.8.1091

2. Wolfensberger B, Ferri R, Bianco G, et al. From physiological neck myoclonus to sleep related head jerk. J Sleep Res. 2019;28:e12831. doi:10.1111/jsr.12831

3. Lopez R, Chenini S, Barateau L, et al. Sleep-related head jerks: toward a new movement disorder. Sleep. 2021;1:44. doi:10.1093/ sleep/zsaa 165

4. Sateia MJ. International Classification of Sleep Disorders-Third Edition. Chest. 2014;146:1387-1394. doi:10.1378/chest.14-0970

5. Luciani BD, Desmet DM, Alkayyali AA, Leonardis JM, Lipps DB. Identifying the mechanical and neural properties of the sternocleidomastoid muscles. J Appl Physiol. 2018;124:1297-1303. doi:10.1152/ japplphysiol.00892.2017

6. Kumar S, Narayan Y, Amell T. Spectral profile of superficial cervical muscles. J Electromyogr Kinesiol. 2001;11:269-280. doi:10.1016/ S1050-6411(01)00007-4

7. Fisher RS, Acevedo C, Arzimanoglou A, et al. ILAE official report: a practical clinical definition of epilepsy. Epilepsia. 2014;55:475-482. doi:10.1111/epi.12550

8. Fisher RS, Cross JH, French JA, et al. Operational classification of seizure types by the International League Against Epilepsy: position Paper of the ILAE Commission for Classification and Terminology. Epilepsia. 2017;58:522-530. doi:10.1111/epi.13670

9. Arshadi R, Ghasemi GA, Samadi H. Effects of an 8-week selective corrective exercises program on electromyography activity of scapular and neck muscles in persons with upper crossed syndrome: randomized controlled trial. Phys Ther Sport. 2019;37:113-119. doi:10.1016/j.ptsp.2019.03.008

10. Iber CA, Chesson A, Quan S. The AASM Manual for the Scoring of Sleep and Associated Events: Rules. 1st ed. Terminology and Technical Specifications; 2007. 
11. Prihodova I, Skibova J, Nevsimalova S. Sleep-related rhythmic movements and rhythmic movement disorder beyond early childhood. Sleep Med. 2019;64:112-115. doi:10.1016/j. sleep.2019.05.021

12. Gogo E, van Sluijs RM, Cheung T, et al. Objectively confirmed prevalence of sleep-related rhythmic movement disorder in pre-school children. Sleep Med. 2019;53:16-21. doi:10.1016/j. sleep.2018.08.021

13. Ferri R, Rundo F, Zucconi M, et al. Putting the periodicity back into the periodic leg movement index: an alternative data-driven algorithm for the computation of this index during sleep and wakefulness. Sleep Med. 2015;16:1229-1235. doi:10.1016/j.sleep.2015.05.019

14. Zutt R, van Egmond ME, Elting JW, et al. A novel diagnostic approach to patients with myoclonus. Nat Rev Neurol. 2015;11:687-697. doi:10.1038/nrneurol.2015.198
15. Apartis E, Vercueil L. To jerk or not to jerk: a clinical pathophysiology of myoclonus. Rev Neurol. 2016;172:465-476. doi:10.1016/j. neurol.2016.07.013

16. Brooks PL, Peever JH. Impaired GABA and glycine transmission triggers cardinal features of rapid eye movement sleep behavior disorder in mice. J Neurosci. 2011;31:7111-7121. doi:10.1523/ JNEUROSCI.0347-11.2011

17. Manconi M, Ferri R, Zucconi M, et al. Dissociation of periodic leg movements from arousals in restless legs syndrome. Ann Neurol. 2012;71:834-844. doi:10.1002/ana.23565

18. Matsumoto RR, Truong DD, Nguyen KD, et al. Involvement of GABA(A) receptors in myoclonus. Mov Disord. 2000;15(Suppl 1):47-52. doi:10.1002/mds.870150709

\section{Publish your work in this journal}

Nature and Science of Sleep is an international, peer-reviewed, open access journal covering all aspects of sleep science and sleep medicine, including the neurophysiology and functions of sleep, the genetics of sleep, sleep and society, biological rhythms, dreaming, sleep disorders and therapy, and strategies to optimize healthy sleep.
The manuscript management system is completely online and includes a very quick and fair peer-review system, which is all easy to use. Visit http://www.dovepress.com/testimonials.php to read real quotes from published authors. 